\title{
Significant effects of Ganoderma lucidum polysaccharide on lipid metabolism in diabetes may be associated with the activation of the FAM3C-HSF1-CAM signaling pathway
}

\author{
RUI PAN ${ }^{*}$, JIAN LOU $^{1 *}$ and LING WEI ${ }^{2}$ \\ ${ }^{1}$ Department of Nutrition, Yuxi People's Hospital of Kunming, Yuxi, Yunnan 653100; ${ }^{2}$ Department of Clinical Nutrition, \\ Second People's Hospital of Yunnan Province, Kunming, Yunnan 650051, P.R. China
}

Received October 20, 2019; Accepted October 16, 2020

DOI: $10.3892 /$ etm.2021.10252

\begin{abstract}
Diabetes is a threat to patient health worldwide. Type 2 diabetes (T2DM), one of the two main types of diabetes, is a long-term metabolic disease caused by heredity and environmental factors. It has been reported that Ganoderma lucidum polysaccharide (GLP) significantly decreased the concentration of blood glucose, promoted insulin secretion, improved glucose tolerance and regulated the concentration of blood lipids. In the present study, a T2DM model was established in $\mathrm{db} / \mathrm{db}$ mice, following which T2DM mice were treated with GLP (100 and $400 \mathrm{mg} / \mathrm{kg}$ ) for 8 weeks, with MET used as the positive control. The glycosylated hemoglobin (HbAlc) and fasting blood glucose (FBG) levels, and diabetes-associated clinical chemistry indexes were detected in the blood and serum of each mouse. Hematoxylin and eosin, and oil red $\mathrm{O}$ staining were performed on the livers of each mouse to evaluate the level of liver fat. The expression levels of family with sequence similarity 3 (FAM3C), heat shock factor 1 (HSF1), calmodulin (CaM), AKT and phosphorylated (p)-AKT were detected in the hepatocytes of T2DM mice using reverse transcription-quantitative PCR and western blotting. The results demonstrated that the unbalanced levels of HbAlc, FBG and diabetes-related index in T2DM mice were significantly improved by treatment with GLP. Lipid droplets in the hepatocytes of mice shrank in the GLP groups compared with the model control group. The expression levels
\end{abstract}

Correspondence to: Dr Jian Lou, Department of Nutrition, Yuxi People's Hospital of Kunming, 245 Renmin East Road, Yuxi, Yunnan 653100, P.R. China

E-mail: loujianyn@126.com

Dr Ling Wei, Department of Clinical Nutrition, Second People's Hospital of Yunnan Province, 176 Qingnian Road, Kunming, Yunnan 650051, P.R. China

E-mail: weilingyn@163.com

*Contributed equally

Key words: diabetes mellitus, type 2, Ganoderma lucidum of FAM3C, HSF1, CaM and p-AKT/AKT in the hepatocytes of T2DM mice were significantly increased following treatment with GLP. In conclusion, GLP exerted significant effects on lipid metabolism in diabetes, which may be associated with the activation of the FAM3C-HSF1-CaM signaling pathway.

\section{Introduction}

Diabetes, a debilitating disease that threatens the health of individuals globally, is becoming a public health issue concerning scientists worldwide since $2015(1,2)$. The primary pathological mechanisms underlying the development of diabetes include glycometabolism and lipid metabolism dysregulation $(3,4)$. If phosphorylation of glucose decreases, the signaling pathways of glycolysis, pentose phosphate and tricarboxylic acid cycle will be blocked $(5,6)$. The synthesis of glycogen is reduced and decomposition increases, resulting in a decline of the body's ability to utilize and take up glucose (7-9), leading to a glycometabolism disorder $(10,11)$. When the ability to utilize and take up glucose from fat tissues declines, the concentration of triglycerides in the plasma increases and the synthesis of fat reduces $(7,12)$, resulting in a lipid metabolism disorder (13). The main indexes of lipid metabolism include fatty acid synthetase, acetyl-CoA carboxylase, acyl-CoA oxidase, and carnitine palmityl transferase $(14,15)$.

There are two types of diabetes, type I and II (T2DM). The number of patients with T2DM makes up $80-90 \%$ of all patients with diabetes in Thailand in 2018 (16). T2DM is a long-term disease caused by metabolic disorders as a result of heredity and environmental factors, which results in a functional defect of islet $\beta$ cells and the secretion of insulin $(17,18)$. In this case, the metabolic disorders involve carbohydrates, proteins, fat, electrolytes and water. Currently, treatments for diabetes include hypoglycemic drugs, including insulin, insulin analogues and sulfonylurea drugs (19-21). However, these hypoglycemic drugs can cause numerous side effects, including liver and kidney injury and insulin resistance (22). Certain Traditional Chinese Medicines are being studied as potential therapeutics for diabetes to increase drug efficacy and decrease side effects.

Ganoderma lucidum (G. lucidum) polysaccharide (GLP) is one of the effective ingredients in the mushroom G. lucidum $(23,24)$. It has been reported that GLP significantly 
decreased the concentration of blood glucose, promoted the secretion of insulin, improved glucose tolerance, regulated the concentration of fat in the blood and postponed the development of diabetic complications $(3,25)$. However, the underlying mechanisms remain unknown. Currently, family with sequence similarity 3 (FAM3C), which is composed of FAM3A-D, has been reported to be associated with the regulation of glucometabolism and lipid metabolism $(26,27)$. Chen et al $(28)$ reported that the FAM3C-heat shock factor 1 (HSF1)-calmodulin (CaM) signaling pathway was significantly inhibited in the liver of a streptozotocin (STZ)-induced type I diabetic mouse model. The expression of glycogen was inhibited, and the high concentration of blood glucose of the mice was significantly improved by overexpressing FAM3C and activating the HSF1-CaM-AKT signaling pathway. Furthermore, Zhang et al (27) demonstrated that as a novel hepatocyte factor, FAM3C significantly inhibited the HSF1-CaM-AKT signaling pathway, by which gluconeogenesis and lipogenesis were suppressed.

In the current study, the hypoglycemic and hypolipidemic effects of GLP were investigated in a T2DM db/db mouse model, along with an investigation of the effects of GLP on the FAM3C-HSF1-CaM signaling pathway in order to determine the underlying mechanism of GLP in the lipid metabolism dysregulation in diabetes.

\section{Materials and methods}

Ethics approval. All animal experiments performed in the current study were authorized by the Ethics Committee of Zhejiang Chinese Medical University and carried out according to the guidelines for the Care and Use of Laboratory Animals and to the Principles of Laboratory Animal Care and Protection (approval no. ZSLL-2018-172).

Animals. A total of 24 adult male (age, 6 weeks; weight, 18-24 g) idiopathic T2DM model mice $(\mathrm{db} / \mathrm{db})$ and $6-+/ \mathrm{db}$ male mice were purchased from Vital River Laboratories Co., Ltd.. The housing room was maintained between $20.0-26.1^{\circ} \mathrm{C}$, with an average humidity between $30-70 \%$ and under a 12-h/12-h light/dark cycle. The db/db mice were fed a high-fat diet for 2 weeks and $-+/ \mathrm{db}$ mice were fed an ordinary diet as a control. All the animals had free access to food and water. Following this, $0.08 \mathrm{ml}$ blood was collected from the tail vein at the endpoint to detect the concentration of glucose in the blood. The animals with concentrations of blood glucose $>11.1 \mathrm{mmol} / \mathrm{l}$ were selected as T2DM model mice.

Preparation of GLP. A total of $\sim 5 \mathrm{~g}$ sporoderm-broken spores of G. lucidum were settled into $100 \mathrm{ml}$ ultrapure water. The lipids were removed and centrifuged at a speed of $1,000 \mathrm{x} g$ at $70^{\circ} \mathrm{C}$ for $12 \mathrm{~h}$. Following this, the mixture was centrifuged at $1,200 \mathrm{x} \mathrm{g}$ for $15 \mathrm{~min}$ to remove the insoluble substances and purified using the Sevage method (29). The solution was then centrifuged $\left(300 \mathrm{x} \mathrm{g}, 10 \mathrm{~min}, 4^{\circ} \mathrm{C}\right)$ to collect the supernatant. Finally, the supernatant was centrifuged $\left(300 \mathrm{x} \mathrm{g}, 10 \mathrm{~min}, 4^{\circ} \mathrm{C}\right)$ again and freeze-dried using a $\mathrm{H} 051$ freeze dryer (LaboGene) to obtain GLP for subsequent experiments.

Groups and dosing. The animals were divided into five groups. The $6-+/ \mathrm{db}$ male mice were used as the wild-type group. The other 24 T2DM model mice were divided into the following groups: i) Model control group; ii) low dose GLP group (GLP $100 \mathrm{mg} / \mathrm{kg} /$ day); iii) high dose GLP group (GLP $400 \mathrm{mg} / \mathrm{kg} /$ day); and iii) melbine (MET, Tianjin Zhongxin Pharmaceutical Group Co., Ltd.) group (MET 300 mg/kg/day, which was used as the positive control). A total of six mice were allocated per group. GLP was dissolved in $0.5 \%$ sodium carboxyl methyl cellulose (Shandong Senmei Biological Technology Co., Ltd.) and MET was dissolved in water. Each animal was dosed by oral gavage every day for 8 weeks and the daily dose volume was adjusted according to the body weight of each mouse. Animal health and behavior were monitored every day.

Glycosylated hemoglobin and clinical chemistry analysis. Following 8 weeks of treatment, levels of glycosylated hemoglobin (HbAlc) were measured with an analyzer (Roche Diagnostics) using whole blood obtained from the tail vein (0.08 ml/animal) from anesthetized mice (1-2\% inhalant isoflurane). Blood $(\sim 0.08 \mathrm{ml})$ was collected from the tail vein of each mouse. Blood was centrifuged at $1,000 \mathrm{x} \mathrm{g}$ at $4^{\circ} \mathrm{C}$ for $15 \mathrm{~min}$. The serum was collected and stored at $-20^{\circ} \mathrm{C}$. Following this, the concentrations of fasting blood glucose (FBG), alanine aminotransferase (ALT), total cholesterol (TC), glutamine transaminase (TG), high-density lipoprotein cholesterol (HDL-C), low-density lipoprotein cholesterol (LDL-C), serum creatinine (Scr) and urea nitrogen (BUN) serum levels were detected using a 7020 full automatic biochemical analyzer (Hitachi, Ltd.). The concentrations of uric acid (UA), uric creatinine (Ucr) and urine microalbumin (U-LAB) in the urine of mice were detected by an automatic biochemical analyzer (Automatic Biochemical Analyzer 7180; Hitachi, Ltd.).

Hematoxylin and eosin $(H \& E)$ staining. Following dosing for 8 weeks, animals were sacrificed by $20-25 \% \mathrm{CO}_{2}$ euthanasia. Following this the veterinarian verified death based on respiratory, heartbeat, pupil and nerve reflex indicators. The liver of each mouse was removed and placed into a plate filled with pre-cooled normal saline. After tissues were fixed in $4 \%$ paraformaldehyde at room temperature for $15 \mathrm{~min}$, they were dehydrated in 50, 70, 80, 95, 100 and 100\% alcohol, for 20 min each time. Subsequently, the dehydrated tissue was placed in the following solutions: Ethanol + xylene (1:1) for $2 \mathrm{~h}$, xylene I and II for $10 \mathrm{~min}$ each, and then placed in xylene + paraffin (1:1) for $2 \mathrm{~h}$, paraffin I $(1 \mathrm{~h})$ and paraffin II $(2 \mathrm{~h})$ to immerse. After embedding in an ice box, 4- $\mu \mathrm{m}$ paraffin slices were made, which were then dewaxed with xylene and gradient alcohol, and stained with hematoxylin and eosin at room temperature for $30 \mathrm{~min}$ for observation under a light microscope (x400 magnification; Olympus Corporation).

Oil Red $O$ staining. The liver of each mouse was frozen at $-20^{\circ} \mathrm{C}$ and cut into $5-10 \mu \mathrm{m}$ sections. The slides were air dried for 30-60 $\mathrm{min}$ at room temperature and fixed in ice cold $10 \%$ formalin for $5-10 \mathrm{~min}$ at $4^{\circ} \mathrm{C}$. Following this, the slides were air dried again for another 30-60 min and placed in absolute propylene glycol for 2-5 min to avoid carrying water into the Oil Red O. The slides were stained in pre-warmed Oil Red O solution for $8-10 \mathrm{~min}$ in a $60^{\circ} \mathrm{C}$ oven and differentiated in $85 \%$ propylene glycol for $2-5 \mathrm{~min}$. Distilled water were used to rinse the slides. Then, the slides were stained at room 
temperature in Gill's hematoxylin for $30 \mathrm{sec}$ and washed thoroughly in running tap water for 3 min. Lastly, the slides were placed in distilled water. Lipid droplets were observed under a light microscope (x200 magnification; Olympus Corporation).

Immunohistochemistry. Liver tissues were embedded in paraffin (fixed in 4\% paraformaldehyde for $20 \mathrm{~min}$ at room temperature), sectioned (3- $\mu \mathrm{m}$ thickness), blocked for $1 \mathrm{~h}$ with $2 \%$ normal horse serum (Sigma-Aldrich; Merck $\mathrm{KGaA}$ ) at room temperature and incubated with FAM3C antibodies (1:500; cat. no. PA5-83654; Thermo Fisher Scientific, Inc.). Tissues were incubated at $37^{\circ} \mathrm{C}$ for 1-2 $\mathrm{h}$ and washed twice for $5 \mathrm{~min}$ with PBS. A HRP-conjugated antibody (1:2,000; cat. no. 31466; Thermo Fisher Scientific, Inc.) was added and incubated for $30 \mathrm{~min}$ at room temperature. A total of $1 \mathrm{ml} \mathrm{3,3'-diaminobenzidine} \mathrm{(DAB)} \mathrm{Plus} \mathrm{substrate}$ (Sigma-Aldrich; Merck KGaA) was mixed with 1-2 drops of DAB Plus Chromogen (Sigma-Aldrich; Merck KGaA) and pipetted onto the slides. Following incubation for 3-15 min, the slides were washed with water and observed with a fluorescence microscope (x200 magnification; Olympus Corporation).

Reverse transcription-quantitative PCR (RT-qPCR) for miR-34a expression. Following dosing for 8 weeks, animals were sacrificed by $20-25 \% \mathrm{CO}_{2}$ euthanasia. The liver of each mouse was removed, and total RNA was collected from the tissues using an RNA Extraction kit (Takara Bio, Inc.), according to the manufacturer's protocols. Extracted RNA was quantified using a NanoDrop spectrophotometer (NanoDrop Technologies; Thermo Fisher Scientific, Inc.). A cDNA Synthesis kit (Takara Biotechnology Co., Ltd.) was subsequently used to synthesize cDNA according to the manufacturer's instructions. SYBR Premix Ex Taq ${ }^{\mathrm{TM}}$ (Takara Bio, Inc.) with an Applied Bio-Rad CFX96 Sequence Detection system (Applied Biosystems; Thermo Fisher Scientific, Inc.) was used for RT-qPCR. The thermocycling conditions were as follows: Initial denaturation at $92^{\circ} \mathrm{C}$ for $4 \mathrm{~min}$, followed by 40 cycles of $90^{\circ} \mathrm{C}$ for $15 \mathrm{sec}$ and $60^{\circ} \mathrm{C}$ for $30 \mathrm{sec}$. The expression levels of FAM3C, HSF1 and CaM were determined by the threshold cycle and relative expression levels were calculated using the $2^{-\Delta \Delta \mathrm{Cq}}$ method (30). The expression of GAPDH in the tissues was used as the negative control. Three independent assays were performed. The primers for each protein are presented in Table I.

Western blotting. The livers were isolated from the animals following dosing for 8 weeks. A Nuclear and Cytoplasmic Protein Extraction kit (Beyotime Institute of Biotechnology) was used to isolate proteins from tissues. Protein concentration was determined using a BCA kit (Guangzhou Youdi Biotechnology Co., Ltd.). Following this, $\sim 30 \mu \mathrm{g} /$ lane of protein was separated via SDS-PAGE on a $12 \%$ gel, and subsequently transferred to a PVDF membrane (EMD Millipore). The membrane was blocked with $5 \%$ non-fat dry milk in Tris-buffered saline with $0.1 \%$ Tween-20 ( $\mathrm{pH} \mathrm{7.4)} \mathrm{for} 1 \mathrm{~h}$ at room temperature, and then incubated overnight at room temperature with primary rabbit anti-human antibodies for FAM3C (1:1,000; cat. no. ab56065; Abcam), HSF1 (1:1,000; cat. no. ab61382; Abcam), CaM (1:1,000; cat. no. ab52476; Abcam), phosphorylated (p)-AKT (1:1,000; cat. no. ab38449; Abcam), AKT (1:1,000; cat. no. ab18785; Abcam) and GAPDH (1:1,000; cat. no. ab8245; Abcam) purchased from Abcam. Membranes were then
Table I. Sequences of primers for FAM3C, HSF1, CaM and GAPDH.

Primer

Primer Sequences (5'-3') length (bp)

FAM3C F: CCACCACAGAAGACCCAGTT 20

R: AACCAAACTCACGGATGAGG 20

HSF1 F: CCAGCAGCAAAAAGTTGTCA 20

R: CTGGTGACAGCATCAGAGGA 20

CaM F: GTTTGGGTGTGTGACTCTGG 21

R: GTTCTGTGAAATCTTCCGGG 20

GAPDH F: CAATGACCCCTTCATTGACC 20 R: GAGAAGCTTCCCGTTCTCAG 20

F, forward; R, reverse; FAM3C, family with sequence similarity 3; HSF1, heat shock factor 1; CaM, calmodulin; bp, base pair.

incubated with HRP-conjugated antibody against rabbit IgG (1:5,000; cat. no. ab6721; Abcam) at room temperature for $2 \mathrm{~h}$. Blots were incubated with enhanced chemiluminescence reagent (Beyotime Institute of Biotechnology) and exposed on a Tanon 5200 Chemiluminescent Imaging System (Tanon Science \& Technology Co., Ltd.) to detect protein bands. Three independent assays were performed. Protein bands were scanned and quantified using a ChemiDoc MP Image analysis system (cat. no. 170-8280; Bio-Rad Laboratories, Inc.).

Statistical analysis. Statistically significant differences for continuous variables were determined using a one-way ANOVA followed by a Dunnett's post hoc test for normally distributed data. All testing was performed using GraphPad Prism software (version 5; GraphPad Software, Inc.). P $<0.05$ was considered to indicate a statistically significant difference. Three independent assays were performed on each assay type.

\section{Results}

Body weight and food intake of T2DM mice are influenced by GLP. The body weight of each mouse was recorded following 8 weeks of dosing. As presented in Fig. 1A, the body weights of the mice in the wild-type $(\mathrm{P}<0.001), 100 \mathrm{mg} / \mathrm{kg}$ GLP $(\mathrm{P}<0.001), 400 \mathrm{mg} / \mathrm{kg}$ GLP $(\mathrm{P}<0.001)$ and MET $(\mathrm{P}<0.01)$ groups were all significantly lower compared with the model control group. The results of food and water intake are presented in Fig. 1B and C. Mice in the wild-type $(\mathrm{P}<0.001)$, $100 \mathrm{mg} / \mathrm{kg}$ GLP $(\mathrm{P}<0.001), 400 \mathrm{mg} / \mathrm{kg}$ GLP $(\mathrm{P}<0.001)$ and MET $(\mathrm{P}<0.001)$ groups consumed significantly less food and water compared with the model control group.

Effects of GLP on the concentration of FBG and HbAlc levels of T2DM mice. The concentration of FBG was detected from tail vein blood samples of T2DM mice. As demonstrated by Fig. 2A, the concentration of FBG in the wild-type $(\mathrm{P}<0.001)$, $100 \mathrm{mg} / \mathrm{kg}$ GLP $(\mathrm{P}<0.01), 400 \mathrm{mg} / \mathrm{kg}$ GLP $(\mathrm{P}<0.001)$ and MET $(\mathrm{P}<0.001)$ groups were all significantly lower compared with the model control group. Furthermore, the level of FBG 

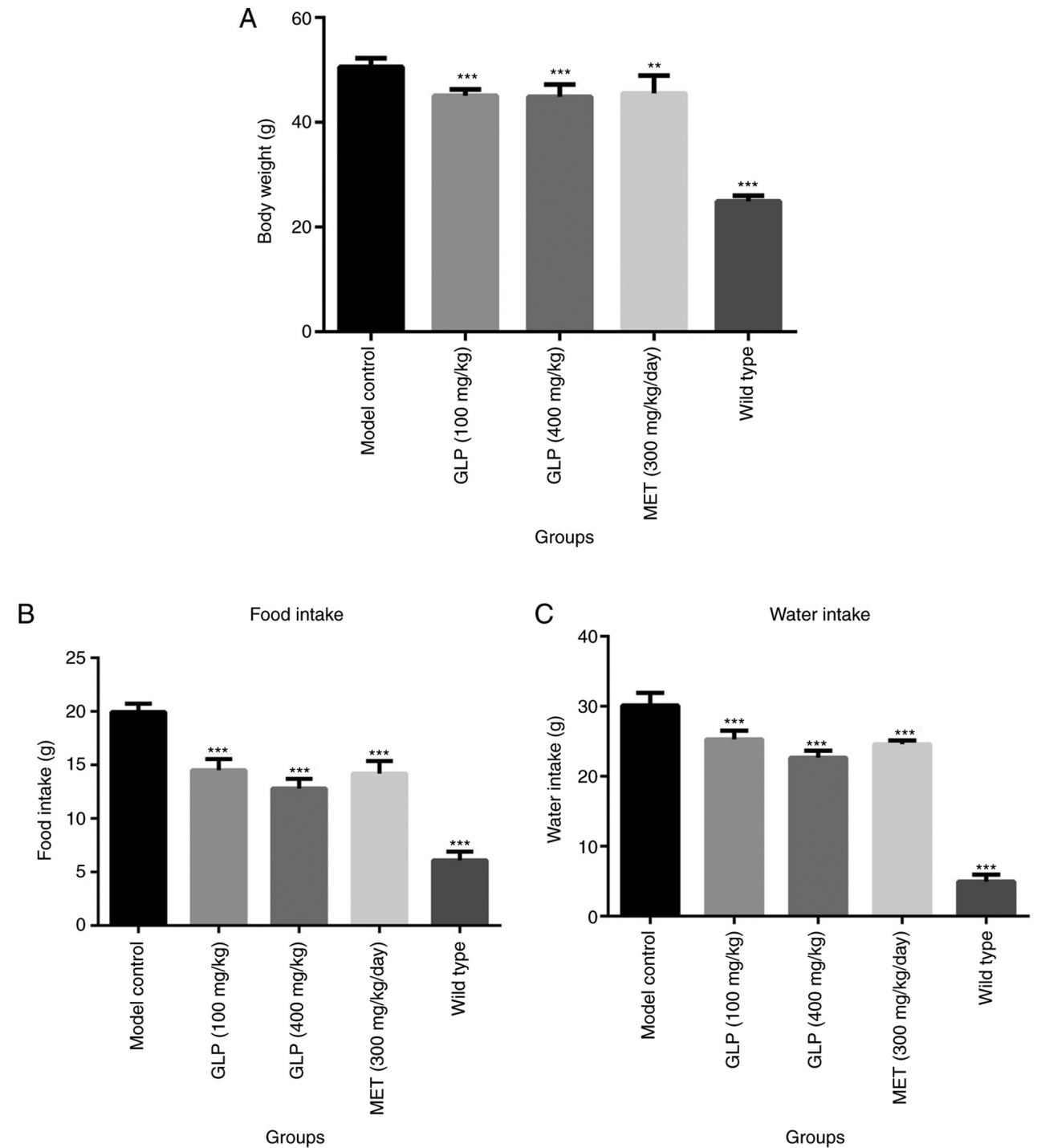

Figure 1. Obese state of type 2 diabetic mice was improved by GLP. (A) Body weight, (B) food intake and (C) water intake of each mouse. ${ }^{* *} \mathrm{P}<0.01$ and ${ }^{* * *} \mathrm{P}<0.001$ vs. model control. GLP, Ganoderma lucidum polysaccharide; MET, melbine.

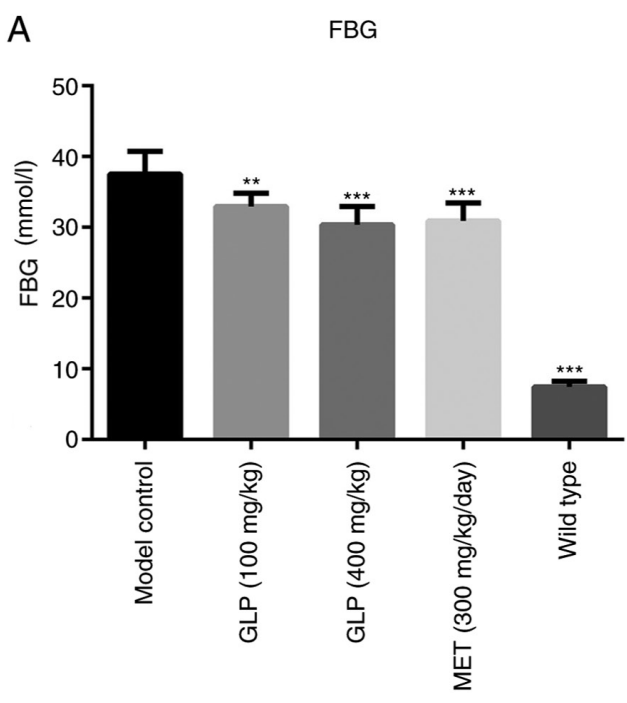

Groups

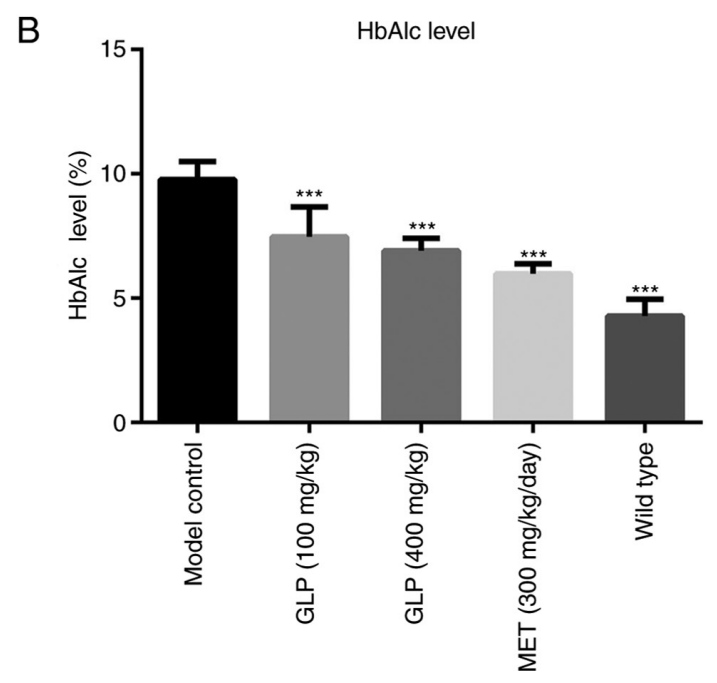

Groups

Figure 2. Effects of GLP on FBG concentration and HbAlc levels of type 2 diabetic mice. Concentrations of (A) FBG and (B) HbAlc in the serum of each mouse. ${ }^{* *} \mathrm{P}<0.01$ and ${ }^{* * *} \mathrm{P}<0.001$ vs. model control. FBG, fasting blood glucose; HbAlc, glycosylated hemoglobin; GLP, Ganoderma lucidum polysaccharide; MET, melbine. 

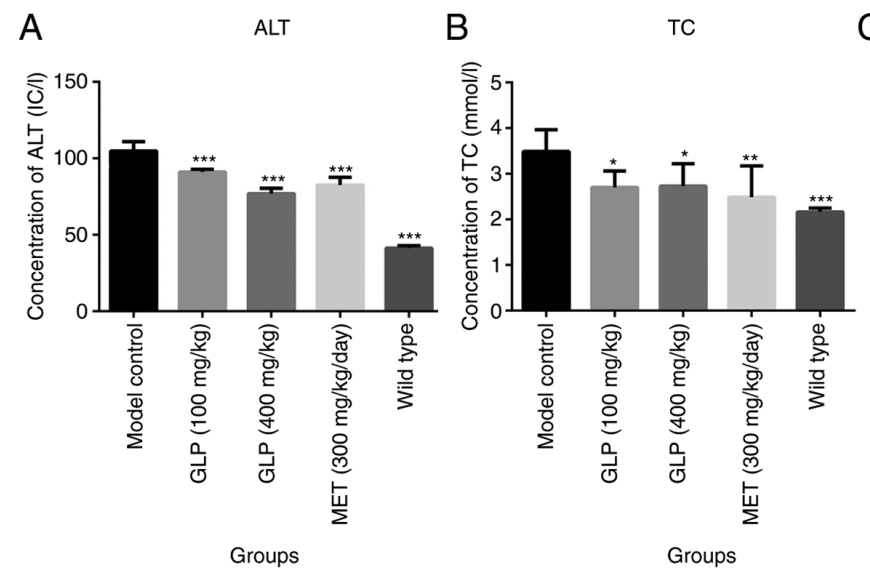

C TG
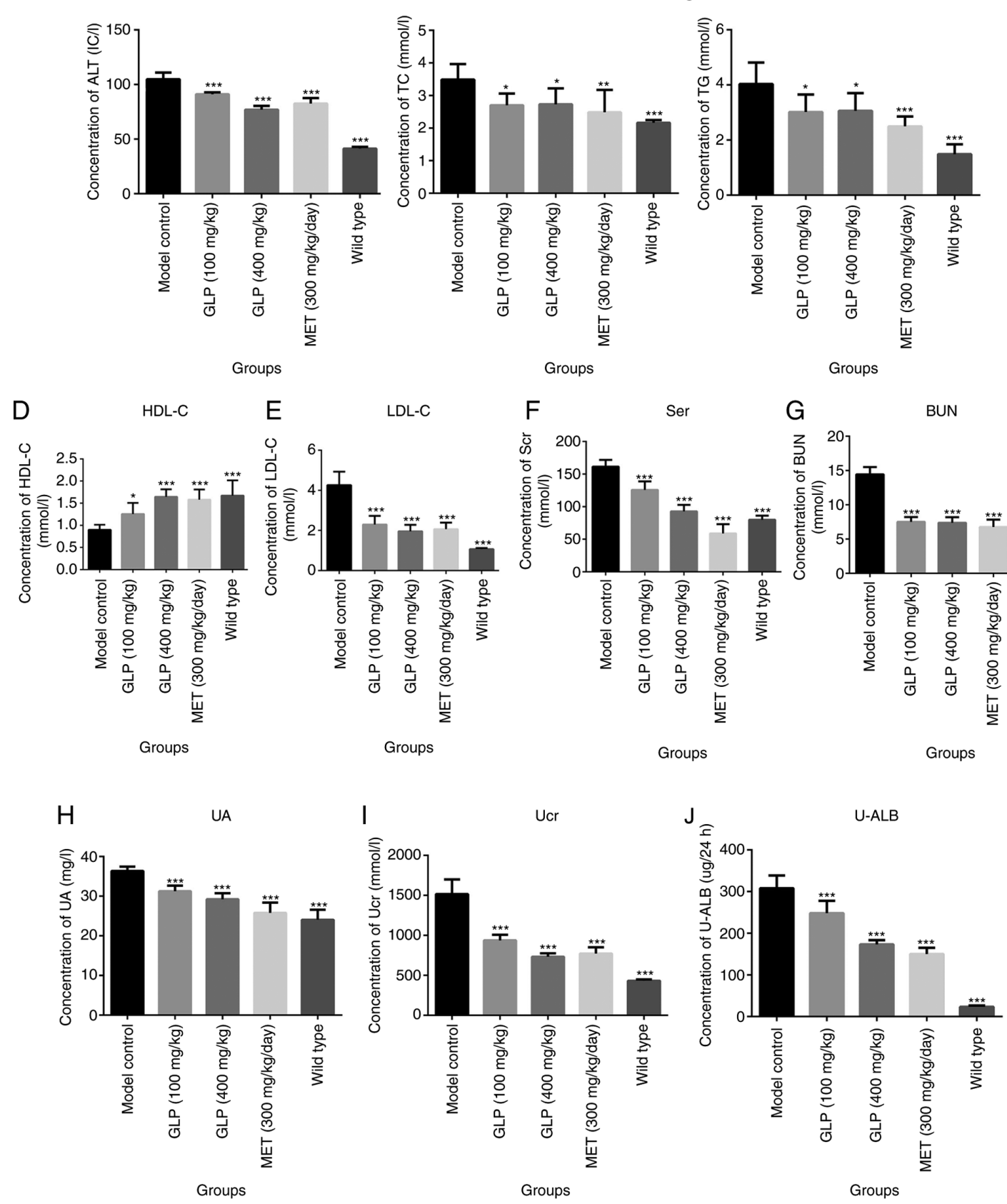

Figure 3. Clinical chemistry of type 2 diabetic mice is altered by GLP. Concentrations of (A) ALT, (B) TC, (C) TG, (D) HDL-C, (E) LDL-C, (F) Scr, (G) BUN, (H) UA, (I) Ucr and (J) U-LAB. ${ }^{*} \mathrm{P}<0.05,{ }^{* * *} \mathrm{P}<0.01$ and ${ }^{* * * *} \mathrm{P}<0.001$ vs. model control. ALT, alanine aminotransferase; TC, total cholesterol; TG, glutamine transaminase; HDL-C, high-density lipoprotein cholesterol; LDL-C, low-density lipoprotein cholesterol; Scr, serum creatinine; BUN, urea nitrogen; UA, uric acid; Ucr, uric creatinine; U-LAB, urine microalbumin; GLP, Ganoderma lucidum polysaccharide; MET, melbine.

in the $400 \mathrm{mg} / \mathrm{kg}$ GLP group was slightly lower compared with the MET group. As presented in Fig. 2B, the HbAlc levels in the wild-type $(\mathrm{P}<0.001)$, GLP $(\mathrm{P}<0.001)$ and MET groups $(\mathrm{P}<0.001)$ were significantly lower compared with the model control group. Additionally, mice in the $400 \mathrm{mg} / \mathrm{kg} \mathrm{GLP}$ group exhibited similar HbAlc levels to the MET group.

Clinical chemistry of T2DM mice is altered by GLP. Following collection of the blood samples after 8 weeks of treatment, the levels of different clinical chemistry indexes were detected in the serum and urine. As shown in Fig. 3, the concentration of ALT, TC, TG, LDL-C, Scr and BUN in the serum of mice in the wild-type $(\mathrm{P}<0.001)$, GLP $(\mathrm{P}<0.01)$ and MET groups $(\mathrm{P}<0.01)$ were significantly lower compared with the model control group. Additionally, the concentration of HDL-C in the serum of mice in the wild-type $(\mathrm{P}<0.001)$, GLP $(\mathrm{P}<0.001$ or $\mathrm{P}<0.05)$ and MET groups $(\mathrm{P}<0.01)$ was significantly higher compared with the model control group. The levels of UA, Ucr and U-LAB in the urine of mice in wild-type $(\mathrm{P}<0.001)$, GLP $(\mathrm{P}<0.001)$ and MET groups $(\mathrm{P}<0.01)$ were significantly lower compared with the model control group.

Hepatic steatosis in T2DM mice is improved by GLP. H\&E and Oil Red O staining were performed to evaluate the effects of 
A

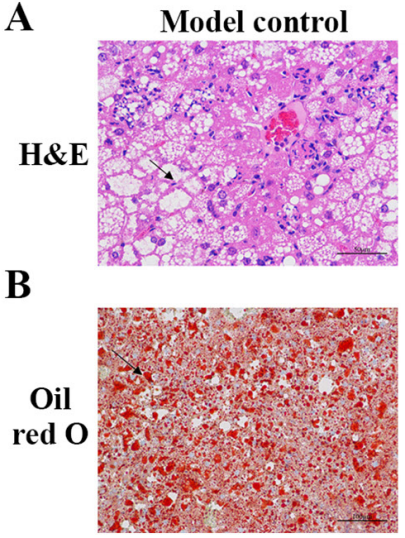

GLP $(100 \mathrm{mg} / \mathrm{kg})$
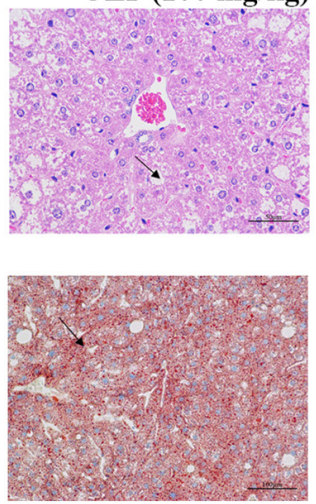

$\operatorname{GLP}(400 \mathrm{mg} / \mathrm{kg})$
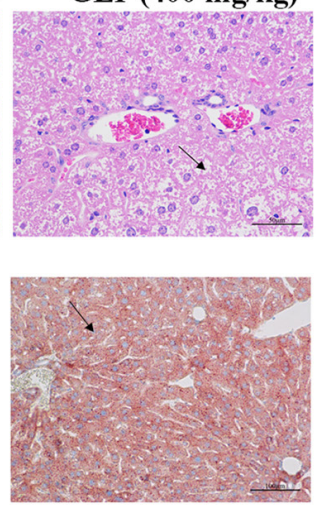
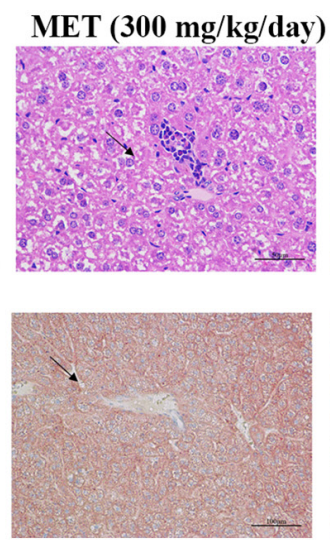

Wild type
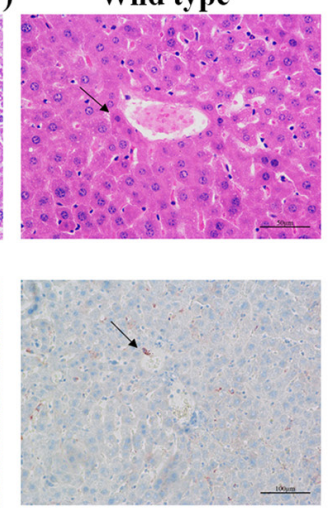

Figure 4. Hepatic steatosis in type 2 diabetic mice is improved by GLP. (A) H\&E staining (magnification, $\mathrm{x} 400$ ) and (B) Oil Red O staining (magnification, $\mathrm{x} 200$ ) of the liver of mice in the model control, $100 \mathrm{mg} / \mathrm{kg}$ GLP, $400 \mathrm{mg} / \mathrm{kg} \mathrm{GLP}, 300 \mathrm{mg} / \mathrm{kg}$ MET and wild-type groups. Arrows indicate lipid droplet vacuoles. H\&E, hematoxylin and eosin; GLP, Ganoderma lucidum polysaccharide; MET, melbine.

A

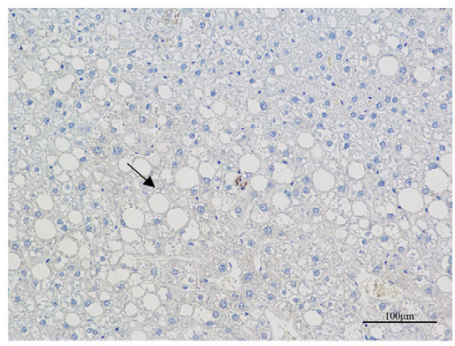

Model control

\section{C}

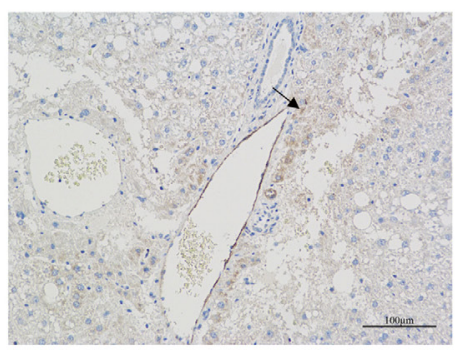

GLP (400 mg/kg)

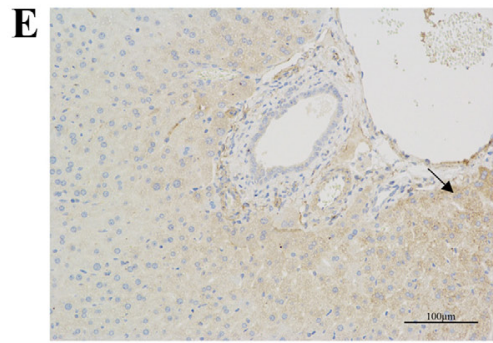

Wild type
B

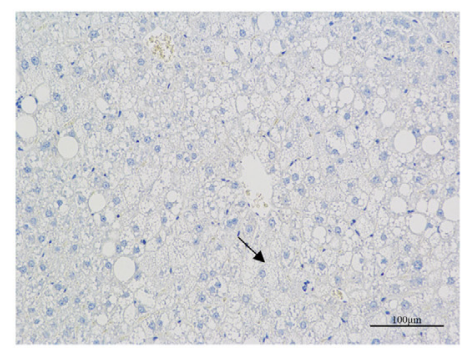

GLP (100 mg/kg)

D

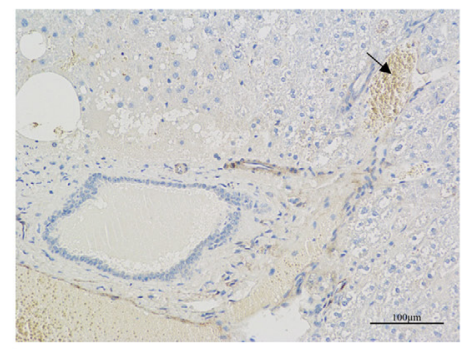

MET (300 mg/kg/day) 

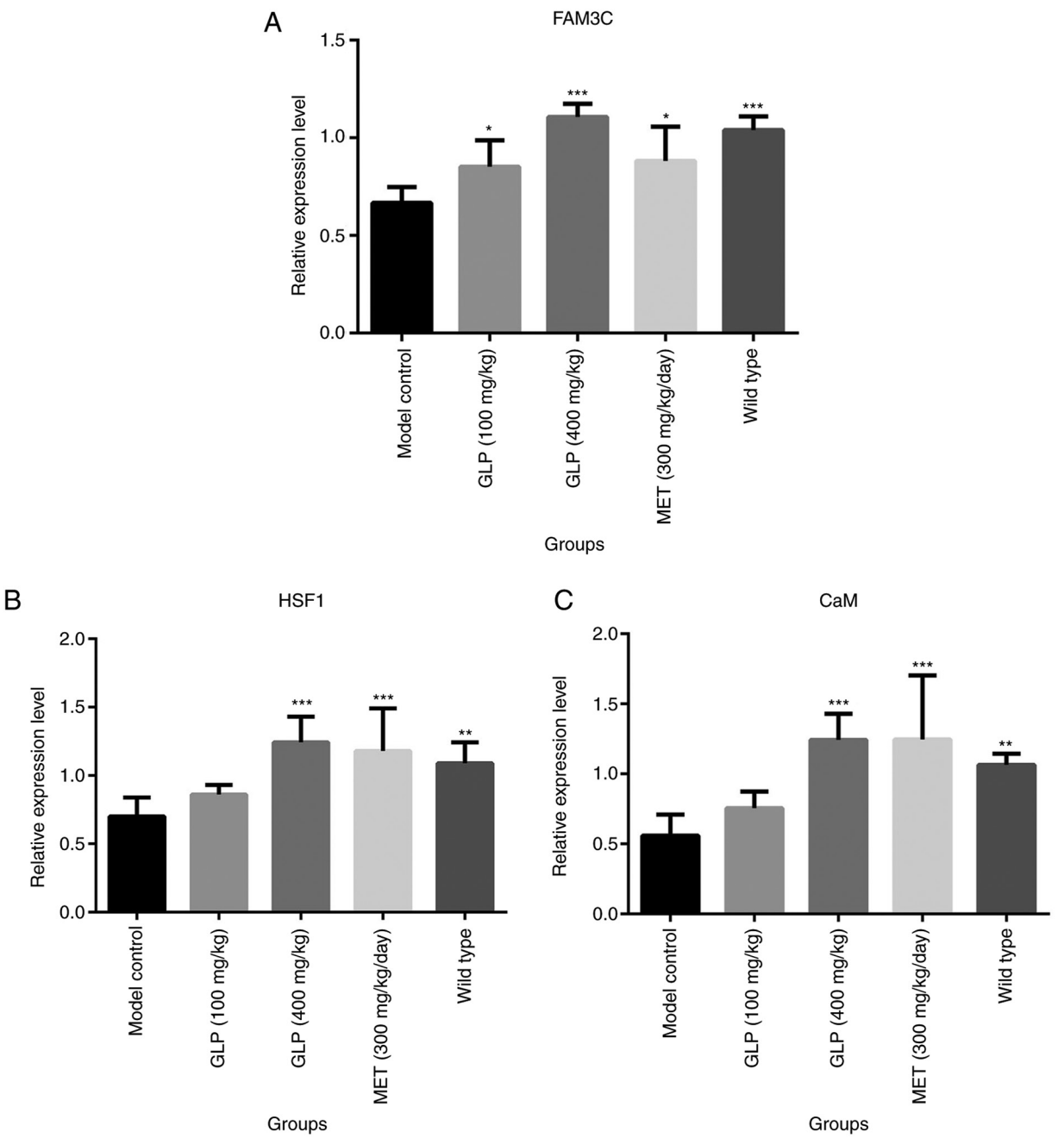

Figure 6. Effects of GLP on the expression of FAM3C, HSF1, and CaM in liver tissues. Relative expression levels of (A) FAM3C, (B) HSF1 and (C) CaM for each group, as determined by reverse transcription-quantitative PCR. ${ }^{*} \mathrm{P}<0.05,{ }^{* * *} \mathrm{P}<0.01$ and ${ }^{* * * *} \mathrm{P}<0.001$ vs. model control. FAM3C, family with sequence similarity 3; HSF1, heat shock factor 1; CaM, calmodulin; GLP, Ganoderma lucidum polysaccharide; MET, melbine.

images, brown indicated FAM3C expression. The results indicated that the expression of FAM3C was markedly higher in the livers of mice in the wild-type, $400 \mathrm{mg} / \mathrm{kg}$ GLP and MET groups compared with model control group, with no significant changes observed in the $100 \mathrm{mg} / \mathrm{kg}$ GLP group. Furthermore, the relative gene expression of FAM3C, HSF1 and CaM in the liver of each mouse was detected by RT-qPCR. The relative expression of FAM3C in the liver of mice in the wild-type and the GLP groups $(\mathrm{P}<0.001$ or $\mathrm{P}<0.05)$ was significantly higher compared with the model control group (Fig. 6). Notably, the expression of FAM3C in the $400 \mathrm{mg} / \mathrm{kg}$ GLP group was higher compared with the MET group. Additionally, HSF1 and $\mathrm{CaM}$ were highly expressed in the liver of the $400 \mathrm{mg} / \mathrm{kg}$ GLP $(\mathrm{P}<0.001)$ and MET $(\mathrm{P}<0.001)$ groups compared with the model group. Subsequently, the expression levels of the proteins FAM3C, HSF1, CaM, AKT and p-AKT were detected by western blotting. As demonstrated in Fig. 7, the expression levels of FAM3C, HSF1, CaM and p-AKT/AKT in the liver of mice in the wild-type $(\mathrm{P}<0.001)$, the $400 \mathrm{mg} / \mathrm{kg}$ GLP $(\mathrm{P}<0.001)$ and MET $(\mathrm{P}<0.001)$ groups were significantly higher compared to the model control group, with slightly or no significant changes observed in the 100 and $400 \mathrm{mg} / \mathrm{kg}$ GLP groups.

\section{Discussion}

G. lucidum is a mushroom that is used in Traditional Chinese Medicine to enhance the immune system, regulate blood glucose and control blood pressure (31). GLP was reported to be one of the main ingredients extracted from G. lucidum $(32,33)$. Furthermore, it has been reported that GLP significantly decreased the concentration of blood glucose, promoted insulin secretion, improved glucose tolerance and regulated the concentration of blood lipids (34). The results of the present study demonstrated that following dosing with 100 and $400 \mathrm{mg} / \mathrm{kg}$ GLP, the FBG, HbAlc and certain diabetes-related clinical chemistry indexes (ALT, TC, TG, LDL-C, Scr, BUN, UA, Ucr and U-LAB) in T2DM mice were significantly improved compared with the model control group. These results were consistent with previous reports $(35,36)$. Diabetes-associated symptoms were reported to be significantly improved in diabetic rats by oral dosing with GLP (37). The concentrations of blood glucose and triglyceride and cholesterol in the serum were decreased following dosing for 3 months (38). Additionally, Ma et al (39) reported that blood glucose levels and diabetic-related morbidity were significantly decreased in multiple low-dose STZ-induced 

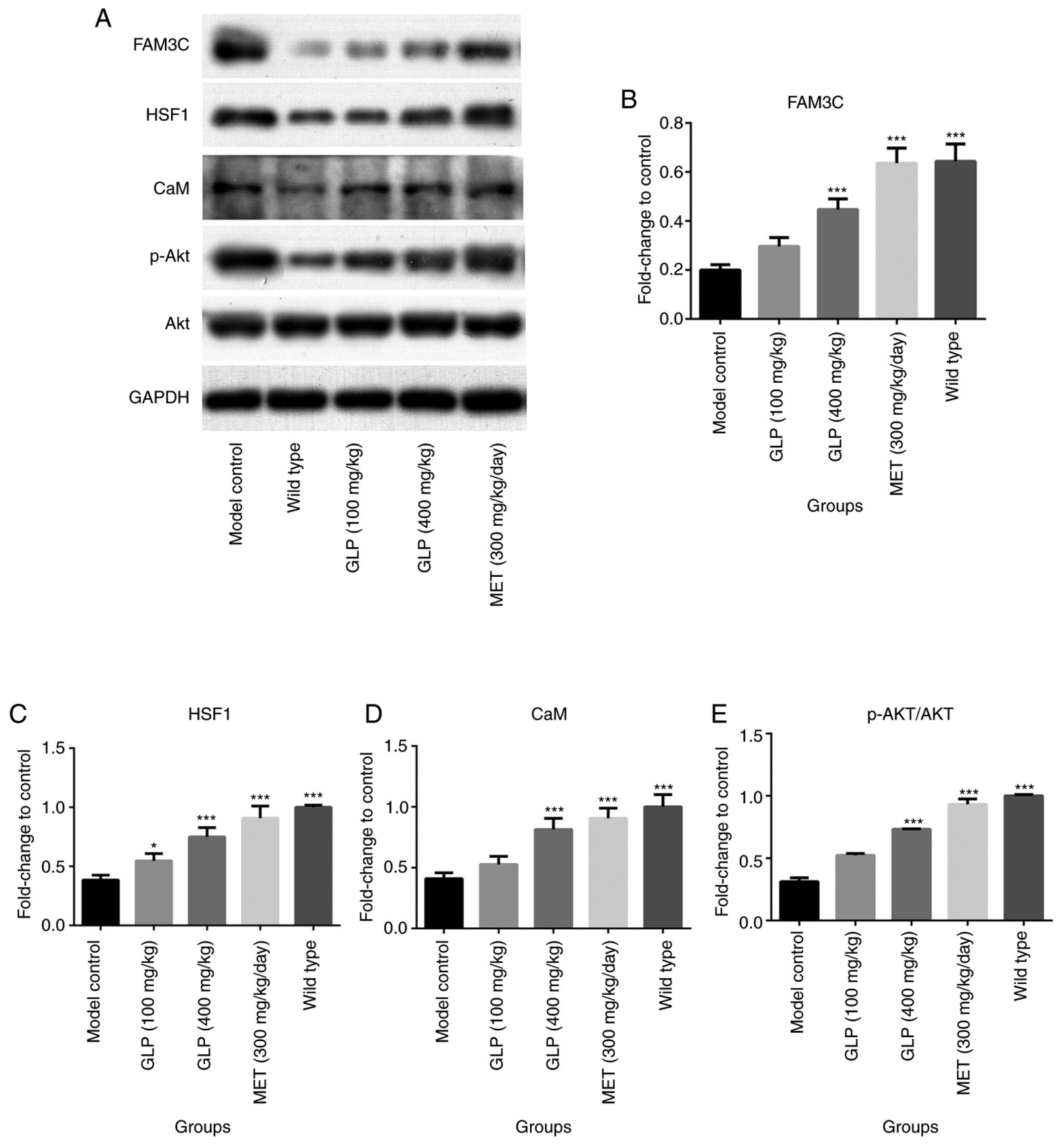

Figure 7. Effects of GLP on the levels of FAM3C, HSF1, CaM, AKT and p-AKT in liver tissues. Expression levels of the proteins (A) FAM3C, HSF1, CaM, AKT and p-AKT in the livers of T2DM mice, as analyzed by western blotting. (B-E) Levels of each protein, as quantified by gray scale analysis. ${ }^{*} \mathrm{P}<0.05$ and ${ }^{* * *} \mathrm{P}<0.001$ vs. model control. FAM3C, family with sequence similarity 3; HSF1, heat shock factor 1; CaM, calmodulin; p-, phosphorylated; GLP, Ganoderma lucidum polysaccharide; MET, melbine.

diabetic mice following dosing with GLP. In the current study, the results of $\mathrm{H} \& \mathrm{E}$ and Oil Red $\mathrm{O}$ staining data demonstrated that the lipid droplets in the hepatocytes of mice shrank in the GLP groups compared with the model control group, which indicated that fatty liver induced by diabetes was significantly improved by GLP treatment. The present study was accompanied with a positive control, MET, which has been used to effectively treat T2DM (40). Notably, nearly all the indexes measured showed that $400 \mathrm{mg} / \mathrm{kg}$ GLP exerted a similar anti-diabetic effect as $300 \mathrm{mg} / \mathrm{kg}$ MET. The current study used a T2DM model to evaluate the anti-diabetic effect of GLP and its effect on the FAM3C-HSF1-CAM signaling pathway. Further verification of the anti-diabetic effects of GLP is being investigated using different types of animal models by the current authors, including GK/IRS-1 double transgenic mice and MKR transgenic mice.

It has been reported that in STZ-induced diabetic mice, the expression of FAM3C is significantly decreased, which results in the inactivation of the HSF1-CaM-AKT signaling pathway. This increases the expression of the glycogen gene, and gluconeogenesis and lipogenesis in the liver become dysregulated, resulting in diabetes $(27,28)$. In the present study, following dosing with GLP for 8 weeks, the expression of FAM3C, HSF1, CaM, AKT and p-AKT in the hepatocytes of mice were detected with immunohistochemistry, RT-qPCR and western blotting. The results indicated that the FAM3C-HSF1-CaM-AKT signaling pathway was significantly activated following GLP treatment in T2DM mice, 
which may explain the significant anti-diabetic effect of GLP. Additionally, the results indicated that MET significantly activated the FAM3C-HSF1-CaM-AKT signaling pathway to exert an anti-diabetic effect. However, based on the current results, it is difficult to ascertain that the effect of GLP is directly associated with FAM3C-HSF1-CaM signaling. Fully understanding the mechanism of the anti-diabetic effect of GLP is an aim of the current authors. Further detailed investigation on the association between the anti-diabetic effects of GLP and the FAM3C-HSF1-CaM signaling pathway should be performed to elucidate the underlying mechanism in the future, including investigations using insulin-resistant in vitro models.

GLPs have previously been demonstrated to exert anti-diabetic effects in multiple reports. Chen et al (23) reported that GLPs effectively lowered blood glucose levels and protected aortas in rats. The underlying mechanism may be involved in the downregulation of advanced glycation end-products and receptor for advanced glycation end-products in aortal tissue. Additionally, it has been reported that GLPs significantly decrease fasting serum glucose levels in T2DM mice in a dose-dependent manner (36). The decrease in fasting serum glucose levels may be associated with decreased mRNA expression level of several key enzymes, such as hexokinase, involved in gluconeogenesis and/or glycogenolysis (41). Furthermore, Xiao et al (42) demonstrated that the anti-diabetic effect of GLPs may be associated with the downregulation of hepatic glucose-regulated enzyme mRNA levels via AMPK activation. However, whether a diabetic-associated signaling pathway was involved in the anti-diabetic effect of GLP remains unknown.

In the present study, the significant anti-diabetic effect of GLPs was verified and the FAM3C-HSF1-CaM signaling pathway was revealed to be associated with this mechanism. Although further, direct verifications are required, these results reported a novel direction for the future investigation of the mechanism of the anti-diabetic effect of GLPs. In conclusion, the present study demonstrated that GLP exerted a significant effect on lipid metabolism dysregulation in diabetic model mice, which may be associated with the activation of the FAM3C-HSF1-CaM signaling pathway.

\section{Acknowledgements}

Not applicable.

\section{Funding}

No funding was received.

\section{Availability of data and materials}

The datasets used and/or analyzed during the current study are available from the corresponding author on reasonable request.

\section{Authors' contributions}

RP was involved in drafting the manuscript and data analysis. JL and LW assisted with the acquisition of data. All authors read and approved the final manuscript.

\section{Ethics approval and consent to participate}

The current study declares that all animal experiments were authorized by the Ethical Committee of Zhejiang Chinese Medical University (Hangzhou, China) and were performed according to the guidelines for the Care and Use of Laboratory Animals and to the Principles of Laboratory Animal Care and Protection.

\section{Patient consent for publication}

Not applicable.

\section{Competing interests}

The authors declare that they have no competing interests.

\section{References}

1. Zhu J, Zhang X, Zhang X, Dong M, Wu J, Dong Y, Chen R, Ding X, Huang C, Zhang Q and Zhou W: The burden of ambient air pollution on years of life lost in Wuxi, China, 2012-2015: A time-series study using a distributed lag non-linear model. Environ Pollut 224: 689-697, 2017.

2. Patil P, Mandal S, Tomar SK and Anand S: Food protein-derived bioactive peptides in management of type 2 diabetes. Eur J Nutr 54: 863-880, 2015.

3. Garber AJ, Abrahamson MJ, Barzilay JI, Blonde L, Bloomgarden ZT, Bush MA, Dagogo-Jack S, DeFronzo RA, Einhorn D, Fonseca VA, et al: Consensus statement by the American Association of clinical endocrinologists and American college of endocrinology on the comprehensive type 2 diabetes management algorithm-2019 executive summary. Endocr Pract 25: 69-100, 2019.

4. He K, Shi JC and Mao XM: Safety and efficacy of acarbose in the treatment of diabetes in Chinese patients. Ther Clin Risk Manag 10: 505-511, 2014.

5. Abe M, Okada K, Maruyama T, Maruyama $\mathrm{N}$ and Matsumoto K: Combination therapy with mitiglinide and voglibose improves glycemic control in type 2 diabetic patients on hemodialysis. Expert Opin Pharmacother 11: 169-176, 2010.

6. Kirschner LS, Qamri Z, Kari S and Ashtekar A: Mouse models of thyroid cancer: A 2015 update. Mol Cell Endocrinol 421: $18-27,2016$

7. Ali MY, Zaib S, Rahman MM, Jannat S, Iqbal J, Park SK and Chang MS: Didymin, a dietary citrus flavonoid exhibits anti-diabetic complications and promotes glucose uptake through the activation of PI3K/Akt signaling pathway in insulin-resistant HepG2 cells. Chem Biol Interact 305: 180-194, 2019.

8. Asano N, Yamashita T, Yasuda K, Ikeda K, Kizu H, Kameda Y, Kato A, Nash RJ, Lee HS and Ryu KS: Polyhydroxylated alkaloids isolated from mulberry trees (Morus alba L.) and silkworms (Bombyx mori L.). J Agric Food Chem 49: 4208-4213, 2001.

9. Chehade JM and Mooradian AD: A rational approach to drug therapy of type 2 diabetes mellitus. Drugs 60: 95-113, 2000.

10. Lorenzo PI, Juárez-Vicente F, Cobo-Vuilleumier $\mathrm{N}$, Garcia-Dominguez M and Gauthier BR: The diabetes-linked transcription factor PAX4: From gene to functional consequences. Genes (Basel) 8: 101, 2017.

11. Omori K, Kobayashi E, Rawson J, Takahashi M and Mullen Y: Mechanisms of islet damage mediated by pancreas cold ischemia/rewarming. Cryobiology 73: 126-134, 2016.

12. Beattie GM, Leibowitz G, Lopez AD, Levine F and Hayek A: Protection from cell death in cultured human fetal pancreatic cells. Cell Transplant 9: 431-438, 2000.

13. Xue YF, Guo CZ, Hu F, Sun DM, Liu JH and Mao SY: Molecular mechanisms of lipid metabolism disorder in livers of ewes with pregnancy toxemia. Animal 13: 992-999, 2019.

14. Kaneko YK: Development and analysis of novel therapeutic targets to improve pancreatic $\beta$-cell function in type 2 diabetes. Yakugaku Zasshi 136: 1623-1629, 2016 (In Japanese).

15. Langley AK, Suffoletta TJ and Jennings HR: Dipeptidyl peptidase IV inhibitors and the incretin system in type 2 diabetes mellitus. Pharmacotherapy 27: 1163-1180, 2007. 
16. Somtimuang C, Olatunji OJ and Ovatlarnporn C: Evaluation of in vitro $\alpha$-amylase and $\alpha$-glucosidase inhibitory potentials of 14 medicinal plants constituted in thai folk antidiabetic formularies. Chem Biodivers 15: e1800025, 2018.

17. Wu H, Liu J, Lou Q, Liu J, Shen L, Zhang M, Lv X, Gu M and Guo X: Comparative assessment of the efficacy and safety of acarbose and metformin combined with premixed insulin in patients with type 2 diabetes mellitus. Medicine (Baltimore) 96 e7533, 2017.

18. Xu J and Rajaratnam R: Cardiovascular safety of non-insulin pharmacotherapy for type 2 diabetes. Cardiovasc Diabetol 16 $18,2017$.

19. Laoud A, Ferkous F, Maccari L, Maccari G, Saihi Y and Kraim K: Identification of novel nt-MGAM inhibitors for potential treatment of type 2 diabetes: Virtual screening, atom based 3D-QSAR model, docking analysis and ADME study. Comput Biol Chem 72: 122-135, 2018.

20. Men P, Li XT, Tang HL and Zhai SD: Efficacy and safety of saxagliptin in patients with type 2 diabetes: A systematic review and meta-analysis. PLoS One 13: e0197321, 2018.

21. Min SH, Yoon JH, Hahn S and Cho YM: Efficacy and safety of combination therapy with an $\alpha$-glucosidase inhibitor and a dipeptidyl peptidase- 4 inhibitor in patients with type 2 diabetes mellitus: A systematic review with meta-analysis. J Diabetes Investig 9: 893-902, 2018

22. Chiefari E, Arcidiacono B, Foti D and Brunetti A: Gestational diabetes mellitus: An updated overview. J Endocrinol Invest 40: 899-909, 2017.

23. Chen Y, Qiao J, Luo J, Wu F, Meng G, Chen H, Zheng H and $\mathrm{Xu} \mathrm{J}$ : Effects of Ganoderma lucidum polysaccharides on advanced glycation end products and receptor of aorta pectoralis in T2DM rats. Zhongguo Zhong Yao Za Zhi 36: 624-627, 2011 (In Chinese).

24. Teng BS, Wang CD, Yang HJ, Wu JS, Zhang D, Zheng M, Fan ZH, Pan D and Zhou P: A protein tyrosine phosphatase 1B activity inhibitor from the fruiting bodies of Ganoderma lucidum (Fr.) Karst and its hypoglycemic potency on streptozotocin-induced type 2 diabetic mice. J Agric Food Chem 59: 6492-6500, 2011.

25. Tamez-Pérez HE, Gonzalez-Guajardo EE and Tamez-Peña AL: Re: Consensus statement by the American association of clinical endocrinologists and American college of endocrinology on the comprehensive type 2 diabetes management algorithm-2019 executive summary. Endocr Pract 25: 622, 2019.

26. Shen L, Ao L, Xu H, Shi J, You D, Yu X, Xu W, Sun J and Wang F: Poor short-term glycemic control in patients with type 2 diabetes impairs the intestinal mucosal barrier: A prospective, single-center, observational study. BMC Endocr Disord 19: 29, 2019.

27. Zhang X, Yang W, Wang J, Meng Y, Guan Y and Yang J: FAM3 gene family: A promising therapeutical target for NAFLD and type 2 diabetes. Metabolism 81: 71-82, 2018.

28. Chen Z, Wang J, Yang W, Chen J, Meng Y, Feng B, Chi Y, Geng B, Zhou Y, Cui Q and Yang J: FAM3C activates HSF1 to suppress hepatic gluconeogenesis and attenuate hyperglycemia of type 1 diabetic mice. Oncotarget 8: 106038-106049, 2017.

29. Zhao X, Li J, Liu Y, Wu D, Cai P and Pan Y: Structural characterization and immunomodulatory activity of a water soluble polysaccharide isolated from Botrychium ternatum. Carbohydr Polym 171: 136-142, 2017.
30. Livak KJ and Schmittgen TD: Analysis of relative gene expression data using real-time quantitative PCR and the 2(-Delta Delta C(T)) method. Methods 25: 402-408, 2001

31. Liu Y, Zhang C, Du J, Jia R, Cao L, Jeney G, Teraoka H, Xu P and Yin G: Protective effect of Ganoderma lucidum polysaccharide against carbon tetrachloride-induced hepatic damage in precision-cut carp liver slices. Fish Physiol Biochem 43: 1209-1221, 2017.

32. Rashad FM, Kattan MHE, Fathy HM, El-Fattah DAA, Tohamy ME and Farahat AA: Recycling of agro-wastes for Ganoderma lucidum mushroom production and Ganoderma post mushroom substrate as soil amendment. Waste Manag 88: 147-159, 2019.

33. Wan Y, Wang Q and Prud'homme GJ: GABAergic system in the endocrine pancreas: A new target for diabetes treatment. Diabetes Metab Syndr Obes 8: 79-87, 2015.

34. Singh P, Jayaramaiah RH, Agawane SB, Vannuruswamy G, Korwar AM, Anand A, Dhaygude VS, Shaikh ML, Joshi RS, Boppana R, et al: Potential dual role of eugenol in inhibiting advanced glycation end products in diabetes: Proteomic and mechanistic insights. Sci Rep 6: 18798, 2016.

35. Xue H, Qiao J, Meng G, Wu F, Luo J, Chen H, Zheng H and $\mathrm{Xu}$ J: Effect of Ganoderma lucidum polysaccharides on hemodynamic and antioxidation in T2DM rats. Zhongguo Zhong Yao Za Zhi 35: 339-343, 2010 (In Chinese).

36. Li HN, Zhao LL, Zhou DY and Chen DQ: Ganoderma lucidum polysaccharides ameliorates hepatic steatosis and oxidative stress in $\mathrm{db} / \mathrm{db}$ mice via targeting nuclear factor $\mathrm{E} 2$ (erythroid-derived 2)-related factor-2/heme oxygenase-1 (HO-1) pathway. Med Sci Monit 26: e921905, 2020.

37. Chen M, Xiao D, Liu W, Song Y, Zou B, Li L, Li P, Cai Y, Liu D, Liao Q and Xie Z: Intake of Ganoderma lucidum polysaccharides reverses the disturbed gut microbiota and metabolism in type 2 diabetic rats. Int J Biol Macromol 155: 890-902, 2020

38. Chang CJ, Lin CS, Lu CC, Martel J, Ko YF, Ojcius DM, Tseng SF, Wu TR, Chen YY, Young JD and Lai HC: Ganoderma lucidum reduces obesity in mice by modulating the composition of the gut microbiota. Nat Commun 6: 7489, 2015.

39. Ma HT, Hsieh JF and Chen ST: Anti-diabetic effects of Ganoderma lucidum. Phytochemistry 114: 109-113, 2015.

40. Adeyemi WJ, Olayaki LA, Abdussalam TA, Fabiyi TO, Raji TL and Adetunji AA: Co-administration of omega-3 fatty acids and metformin showed more desirable effects than the single therapy on indices of bone mineralisation but not gluco-regulatory and antioxidant markers in diabetic rats. Biomed Pharmacother 121: $109631,2020$.

41. Xiao C, Wu QP, Cai W, Tan JB, Yang XB and Zhang JM: Hypoglycemic effects of Ganoderma lucidum polysaccharides in type 2 diabetic mice. Arch Pharm Res 35: 1793-1801, 2012.

42. Xiao C, Wu Q, Zhang J, Xie Y, Cai W and Tan J: Antidiabetic activity of Ganoderma lucidum polysaccharides F31 down-regulated hepatic glucose regulatory enzymes in diabetic mice. J Ethnopharmacol 196: 47-57, 2017. 